\title{
Percepción de los grandes contribuyentes en torno a la administración tributaria venezolana
}

\author{
José Guillermo García Chourio* \\ Dennys González Hernández**
}

\section{Resumen}

La administración tributaria venezolana ha atravesado por profundas reformas institucionales durante más de una década, periodo en el cual también es posible que hayan sucedido cambios en la percepción que tenían los contribuyentes sobre este sector del Estado. Con base en este supuesto, nuestro trabajo tiene como objetivo identificar las percepciones y expectativas que tiene el contribuyente especial (un tipo particular de contribuyente) en torno a la administración tributaria. A partir de un estudio de campo, que implicó un análisis estadístico sobre una muestra de este tipo de contribuyente, se pudo conocer el desarrollo de una estructura de opinión favorable que contrasta con la tradicional imagen negativa que existía sobre el funcionamiento de esta administración, la cual, no obstante, se ve limitada en ganar mayores niveles de legitimidad debido a que la misma depende en gran parte del uso racional que den los órganos decisorios y ejecutivos del Estado a los recursos obtenidos por la vía de la recaudación de impuestos.

Palabras clave: administración tributaria, contribuyentes especiales, reforma del Estado, modernización administrativa, recaudación fiscal.

\section{Introducción}

T a reforma al sistema tributario venezolano en los últimos tiempos marca un ـambio fundamental en el ámbito administrativo debido especialmente al establecimiento de una tipología de contribuyentes según el criterio del máximo ingreso para un máximo aporte a la recaudación fiscal, aquí es donde surge la categoría contribuyente especial, concepto que viene principalmente a identificar a la gran empresa.

\footnotetext{
* Profesor de posgrado en la especialización en Tributación de la Universidad del Zulia y de la licenciatura de la Universidad Católica Cecilio Acosta, Venezuela. Investigador acreditado en el Programa de Promoción a la Investigación del Ministerio de Ciencia y Tecnología de Venezuela. Correo electrónico: jggarcia@unica.edu.ve **Profesora Titular de La Universidad del Zulia, Venezuela. Correo electrónico: franckbolivar@hotmail.com
} 
La razón que impulsa el desarrollo de dicha categoría ha estado centrada en la búsqueda de una administración eficiente dirigida a controlar y proveer de recursos al fisco a partir de concentrar esfuerzos en aquellos contribuyentes con gran capacidad contributiva. Por mucho tiempo el Estado prescindió de una seria extracción impositiva hacia este tipo de contribuyente, dado los enormes ingresos petroleros que sustentaban la promoción de un modelo económico orientado hacia la demanda.

Durante ese periodo de bonanza, la administración tributaria acuñó una imagen de ineficiencia y corrupción, se convirtió en una institución caracterizada por el inmovilismo e incluso se le llegó a considerar como una infraestructura administrativa que para recaudar y controlar era una verdadera nulidad (Purroy, 1990). No obstante, a partir de la última década del siglo XX, fue evidente el cambio de una administración con escasos mecanismos de control ineficiente, promotora de la elusión y la evasión, por una que se fundamenta en la simplificación de los procedimientos, elevado control fiscal y una atención directa hacia el contribuyente, al cual lo considera como un cliente.

Ante estas transformaciones de las que ha sido objeto la administración tributaria es posible esperar que estén operando cambios en la población general y en particular del sector empresarial en cuanto a la percepción negativa que tradicionalmente se ha tenido sobre esta área del aparato público. En este sentido y con base en sondear la opinión del sector empresarial en el Municipio Maracaibo del estado Zulia, este trabajo tiene como objetivo identificar las percepciones y expectativas que tienen los contribuyentes especiales en torno a la administración tributaria; para ello tomamos en cuenta que dicho ámbito del aparato estatal venezolano ha sido objeto de reformas institucionales de largo aliento que ya alcanzan más de una década, como parte de las transformaciones experimentadas por el Estado con miras a ajustar su funcionamiento al modelo de acumulación centrado en la lógica del mercado.

\section{El gran contribuyente: objetivo de una administración tributaria moderna}

La figura del gran contribuyente dentro de los proyectos de reforma tributaria no es nueva en Venezuela. A pesar de su reciente impulso desde mediados de la pasada década, ya la Comisión de Estudios y Reforma Fiscal (CERF), creada en 1980 a raíz de los primeros síntomas de la crisis fiscal, había considerado la importancia de este tipo de contribuyente dentro de un programa de reforma que para aquel entonces 
estuvo orientado hacia dos componentes fundamentales: 1) proteger el consumo interno a través de la sustitución de importaciones para desarrollar la actividad industrial y comercial interna; y 2) disminuir la dependencia del ingreso fiscal petrolero.

En el renglón de importaciones, el elevado volumen de los derechos de importación reflejaba la gran dependencia del país de la producción exterior, situándose la recaudación por concepto de derechos de importación en el 72\% del producto total de los diferentes impuestos indirectos sobre productos no petroleros; mientras que el ingreso fiscal petrolero, representaba casi el $80 \%$ de los ingresos presupuestarios en 1980 (CERF, 1983); en consecuencia, el ingreso no petrolero constituido para el momento por la recaudación del impuesto sobre la renta de personas jurídicas y naturales, los impuestos a la nómina y la recaudación del arancel de aduana no tenían una representación significativa para el fisco.

En el ámbito administrativo, los planteamientos de la CERF fueron dirigidos a mejorar la organización del sistema tributario sobre la base de criterios de separación de las funciones normativa y operativa, descentralización de funciones y la organización funcional en lugar de organización por impuestos. Con respecto a la relación fisco-contribuyente no se plantearon sistemas especiales para los diferentes contribuyentes; aun cuando para los grandes contribuyentes se reconoció que era posible darles un tratamiento especial debido a la complejidad de las transacciones realizadas, sólo se sugirió la necesidad de contar con fiscales entrenados en sistemas modernos de información y auditorías por computación.

La CERF consideró que en las administraciones regionales una sección podía encargarse de los grandes contribuyentes sin necesidad de que se estableciera formalmente una división de grandes contribuyentes en el nivel operativo. Esto era esperable porque para ese momento el sector empresarial gozaba de un elevado proteccionismo estatal y las obligaciones tributarias de los grandes contribuyentes no constituían un objetivo tributario de gran relevancia. En rigor, lo fundamental para la administración tributaria seguía siendo la recaudación petrolera, a pesar de que su aporte al fisco comenzaba a disminuir cada vez más en el transcurrir de los años.

En la década de 1990 surge un mayor interés por la reforma del sistema tributario con la reestructuración económica basada en el modelo neoliberal, propuesto por organismos multilaterales desde los años de 1980 y apoyada en experiencias de países industrializados. La esencia de los nuevos postulados se basa en la programa- 
ción del libre comercio de bienes y servicios en el ámbito mundial, mediante una reducción sustancial de los aranceles y de la eliminación de todas las barreras no arancelarias que se aplican sobre las importaciones (CIAT, 1996). Sin embargo, no se trata de permitir una reducción fiscal originada para el libre comercio, sino que sea compensada con el ingreso tributario interno. El déficit que se ocasionaría en las recaudaciones tributarias por la disminución de los aranceles sería atenuado con un aumento de los impuestos al consumo y por la eliminación de las exenciones fiscales. Con la nueva política del Estado se crean cambios en la administración tributaria porque, como afirma Tanzi (1996: 87), "las Reformas Tributarias han sido partes integrantes de un conjunto de cambios de política introducidos para estimular la eficiencia de la economía y reducir la carga que le impone el sector público".

Por otra parte, se busca el fortalecimiento del sistema tributario, además de conducirlo hacia el nuevo orden económico, lo que implica la sustitución del tradicional sistema de impuesto basado en el impuesto sobre la renta por formas de impuestos donde toda la sociedad tenga la condición de contribuyente; en opinión de Metcalfe y Richards (1989: 229):

[...] la estrategia de eficiencia no es un fenómeno aislado. Forma parte de una visión más amplia de la economía y de la sociedad y de una estrategia radical de cambio cultural e institucional que tiene por objeto dar una mayor libertad a la iniciativa privada, ampliar la eficiencia de las fuerzas del mercado y depender más del esfuerzo individual frente a los cuidados del Estado niñera.

El cambio planteado se fundamenta en la importancia de las instituciones para promover la eficiencia del Estado como condición sine qua non para un óptimo comportamiento de los mercados. En el informe del Banco Mundial de 1997 se reconoce este señalamiento cuando se afirma que "el establecimiento de las instituciones necesarias para un sector público capaz es indispensable para mejorar la eficacia del Estado" (1997: 91). Una de estas instituciones lo representan los sistemas tributarios, los cuales actúan como mecanismos de regulación del mercado en el ámbito macroeconómico de los países al imponer las regalías públicas que la sociedad está en condiciones de exigir de la acumulación privada de capital.

Es así como en el marco de los procesos de modernización tributaria - iniciados en América Latina a partir de la década de los noventa con el objetivo de lograr la eficiencia de los sistemas tributarios en la región - fue creado en Venezuela en agosto de 1994 el Servicio Nacional de Administración Tributaria (SENIAT). La 
gestión de dicho servicio ha estado centrada en lograr la eficiencia en la recaudación, lo que ha supuesto el desarrollo de estrategias para tal fin, donde la más innovadora es la creación de la figura del contribuyente especial; en consecuencia, se establece en el nivel operativo la División de Contribuyentes Especiales para un control directo de las obligaciones tributarias de dichos contribuyentes.

Cuando surgió el proyecto SENIAT, su base fundamental era la creación de unidades de contribuyentes especiales dentro de la antigua administración de rentas, debido a que crear modelos para la universalidad de contribuyentes exige el empleo de cuantiosos recursos y esfuerzos que, por lo general, no están a la disposición de manera inmediata, pues se requieren cambios de organización, sistemas, procedimientos, capacitación, formación, como también cambios de actitud en los funcionarios fiscales y en los propios contribuyentes con lo que se dificulta el control integral de toda la situación tributaria. Sin embargo, al final se optó por la creación de un servicio autónomo nacional que contara dentro de su organización con un área estratégica dedicada, exclusivamente, a la gestión tributaria en el caso de los grandes contribuyentes.

Por lo que respecta a la División de Contribuyentes Especiales, ésta concentra un reducido número de contribuyentes de elevada influencia económica para el país por tener el mayor peso en la recaudación interna del impuesto; esto permite a la administración tributaria elevar su eficiencia, lo que beneficia a los contribuyentes que realmente tienen la posibilidad de aportar al fisco. En relación con ello, algunas reformas han significado la implementación de modelos de atención, control y trato preferencial hacia este tipo de contribuyente, creándose en los últimos años un gran número de disposiciones legales, normas, resoluciones administrativas, actualización de registros e implementación de sistemas de información con base en las características generales que este actor posee.

La intención de adoptar un sistema de control de los grandes contribuyentes es actuar prioritariamente sobre el conjunto más importante del universo de contribuyentes del sistema tributario, lo cual hace menos costosa y más efectiva la labor de la administración, pues al estar la recaudación concentrada en un limitado número de éstos, sólo se requiere la utilización de poco recurso humano y material - eso sí, altamente capacitado y con herramientas tecnológicas de punta - para el control de un tipo de sujeto económico que se caracteriza por realizar grandes y complejas operaciones, casi siempre relacionadas con el comercio exterior, tarea que se hacía muy difícil en el pasado debido a la inexisten- 
cia de un registro diferenciado de contribuyentes que restaba posibilidades de cobro sobre los montos adeudados por impuestos, sanciones e intereses de las grandes empresas con lo que se afectaban significativamente los niveles de recaudación.

La categoría de contribuyente especial es atribuida dentro del sistema tributario venezolano al tipo de contribuyente, generalmente de naturaleza jurídica, que se caracteriza por altos niveles de renta, por lo cual su contribución al fisco supondría ser mayor que la de un contribuyente ordinario o persona natural. Surgen, así, como características relevantes del contribuyente especial la de ser personas jurídicas que generan elevados ingresos en su actividad empresarial. Según los criterios fijados por el SENIAT para el año 2005, con un valor por Unidad Tributaria (UT) de veintinueve mil cuatrocientos Bolívares (Bs. 29.400,oo), aproximadamente unos 15 dólares americanos, los ingresos mínimos de este tipo de contribuyente están situados en un mínimo de treinta mil unidades (30.000 UT), traducidas de los ingresos anuales obtenidos en el ejercicio fiscal inmediatamente anterior. Esta condición económica es susceptible de ser aplicada básicamente a las personas jurídicas dedicadas a la actividad empresarial por ser el sector donde es más factible obtener mayores ingresos por tributación a diferencia de una persona natural, quien por lo general no alcanza a obtener los ingresos suficientes como para ubicarse dentro de estos elevados rangos de renta.

De esta manera, la figura de contribuyente especial es asociada al empresario, es decir, es la denominación utilizada dentro del sistema tributario para denotar al gran empresario, sector que tradicionalmente dentro de las economías capitalistas ha fundamentado la elaboración de parte de las políticas fiscales del Estado.

En el caso venezolano, la base legal de la figura del contribuyente especial procede del artículo No. 40 del Código Orgánico Tributario de 1994, el cual señala:

La administración tributaria podrá establecer plazos para la presentación de declaraciones juradas y pagos de los tributos, con carácter general para determinados grupos de contribuyentes de similares características cuando razones de eficiencia y costo operativo así lo justifiquen (Congreso de la República, 1994).

Este artículo destaca la distinción en el tratamiento de algunos contribuyentes de acuerdo con ciertas características, que si bien no las define, ya dejaba a potestad de la propia administración tributaria identificar aquellos grupos de contribuyentes que con base en su perfil, permitieran a dicha administración el desarrollo de una gestión eficiente de sus operaciones. 
Este tipo de contribuyente asume mayor claridad en el artículo No. 60 de la Ley de Impuesto al Consumo Suntuario y las Ventas al Mayor, sancionada por el Congreso de la República en 1994, donde de manera tácita se incorpora el carácter de especial para determinados contribuyentes cuando establece que

[...] la Administración Tributaria en uso de las atribuciones que le confiere el artículo 40 del Código Orgánico Tributario, podrá establecer con carácter general, en cada caso, para los contribuyentes y responsables que ella califique como especiales, fechas y lugares de pago singulares, de acuerdo con los criterios objetivos que se precisen por Resolución (El subrayado es nuestro).

En cuanto a este artículo se manifiesta parte del alcance de la condición de especial de ciertos contribuyentes para quienes la administración tributaria brindaría una atención personalizada en relación con ofrecerle lugares y fechas particulares de pago.

Así como Venezuela, en la mayoría de los países latinoamericanos se han diseñado formas de organizar y segmentar al universo de los contribuyentes, donde el mayor porcentaje de la recaudación de las obligaciones tributarias proviene de los contribuyentes especiales. Una afirmación reiterada por algunos organismos multilaterales como el Banco Mundial, el Banco Interamericano de Desarrollo y el Centro Interamericano de Administraciones Tributarias - es que en casi todos los países existe una alta concentración de la recaudación en un pequeño grupo de contribuyentes. En el caso de algunos países de América Latina, generalmente un número reducido de contribuyentes entre 0,35\% y 2\% aportan el 51\% y el 75\% de la recaudación (CIAT, 2004).

En este orden de ideas, la realidad en Venezuela sobre la importancia del contribuyente especial en términos de recaudación es muy significativa. Según cifras de SENIAT en 2005 las rentas internas por concepto de tributación a personas naturales y jurídicas fueron equivalentes a un 8, 6 \% del Producto Interno Bruto, de los cuales el 4,1\% correspondieron a la recaudación proveniente del sector de contribuyente especial, lo que representa un 50\% del total de recaudación (SENIAT, 2006). Los contribuyentes especiales de la Gerencia Regional de Tributos Internos del SENIAT-Región Zuliana forman parte de este escenario, sobre todo por la relevancia que tiene su actividad comercial e industrial en el desarrollo económico, social y político de la nación, debido a que gran parte de la economía regional gira en torno a explotación de petróleo. En sólo tributación interna, el estado Zulia aporta un 0,56\% del PIB de dicha recaudación nacional sin considerar los ingresos aduanales. 


\section{Aspectos operativos del estudio}

El estudio implicó un trabajo de campo en torno a una población de contribuyentes especiales del municipio Maracaibo del estado $\mathrm{Zulia}^{1}$, considerados como una clase dentro de la totalidad de contribuyentes registrados por la Gerencia Regional de Tributos Internos del SENIAT. Dicha población oscila en unos 740 contribuyentes, todos dedicados a las actividades empresariales en la zona metropolitana, fundamentalmente en actividades de comercio, industria y servicios, ligadas muchas de ellas a la actividad petrolera. (SENIAT- División de Contribuyentes Especiales, 2004). En tal sentido, se consideró fundamental ubicar sus expectativas, la percepción que tienen de la administración tributaria, así como sus niveles de satisfacción en su condición de grandes contribuyentes para cumplir voluntariamente con sus obligaciones tributarias.

Para determinar el tamaño de la muestra seguimos a Galtung con el "principio de las utilidades decrecientes"; es decir, no es tan importante el tamaño de la muestra como lo que se gana en términos del nivel de significación. Al efecto, tomamos un nivel de significación del $0.05 \%$. Si tomamos en cuenta la significación de proporciones y aplicamos la fórmula respectiva, obtenemos un tamaño de muestra de 33 individuos (población: 740, muestra: 32,95). Ahora bien, dado que la población está clasificada (industria, comercio, servicios) y el número por tipo de individuos es muy dispar (sólo 59 industrias contra 391 comercios, por ejemplo), se decidió tomar una muestra estratificada no proporcional, de la cual obtenemos once individuos por cada estrato, tal como se muestra en el cuadro 1.

\section{Cuadro 1 \\ Muestreo estratificado no proporcional de contribuyentes especiales del Municipio Maracaibo}

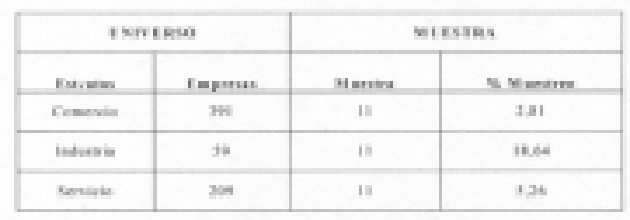

Fuente: Elaboración propia

\footnotetext{
${ }^{1}$ Este municipio abarca territorialmente la mayor extensión $\left(393 \mathrm{Km}^{2}\right)$ de la zona metropolitana de la Ciudad de Maracaibo, segunda ciudad en importancia del país por su tamaño poblacional (más de un millón doscientos mil habitantes, según cifras del Instituto Nacional de Estadísticas, 2005) y nivel de actividad económica. Dicha ciudad viene a ser la capital del estado Zulia, principal estado productor de petróleo, situado en el extremo norte occidental de Venezuela.
} 
Determinado el tamaño de la muestra, se seleccionaron los individuos en cada estrato de manera aleatoria; en primera instancia, se produjo una lista de las empresas o individuos por estrato y se seleccionó en cada uno de los individuos necesarios; posteriormente, se tomaron las muestras mediante el método aleatorio simple; los resultados se exponen en el cuadro 2.

\section{Cuadro 2}

\section{Muestreo aleatorio simple de contribuyentes especiales} del Municipio Maracaibo

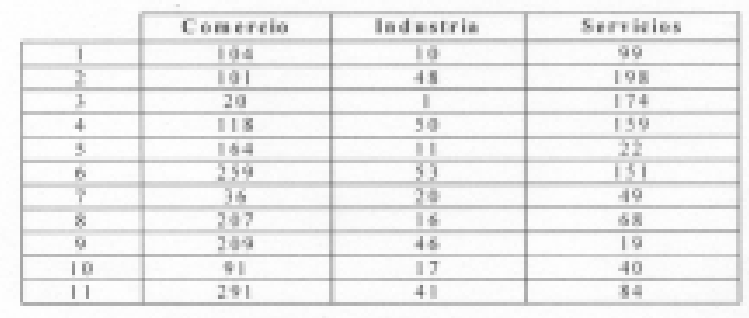

Fuente: Elaboración Propia

Para la medición de las variables: percepción del contribuyente especial y expectativas del contribuyente especial se utilizó un cuestionario estructurado, el cual estaba constituido por veinte ítems, de los cuales siete fueron preguntas abiertas, cinco preguntas cerradas dicotómicas y ocho preguntas de múltiples alternativas, que abarcaban aspectos referidos al funcionamiento de la administración tributaria, experiencia del contribuyente en el cumplimiento de las obligaciones tributarias, mejoramiento de la prestación de servicios públicos, formas de reconocimiento y distinción.

\section{Análisis de los resultados}

Un primer acercamiento a los datos obtenidos permite observar la presencia de un significativo grado de legitimidad en una de las dependencias del Estado, la cual por mucho tiempo estuvo confinada a un gran descrédito por parte de la población. Al parecer, las transformaciones ocurridas en la administración tributaria venezolana en los últimos años han generado signos positivos en la percepción de los contribuyentes; en este sentido, el gráfico 1 hace alusión a la opinión que tiene el contribuyente en torno al SENIAT, ahí 70\% de los encuestados expresa un juicio favorable 
hacia dicho ente, mientras que un 30\% recoge una opinión desfavorable, cifra que — si bien sigue siendo alta - está muy debajo de los elevados niveles de desprestigio social que poseen otras instituciones de la sociedad venezolana.

\section{Gráfico 1 \\ Imagen del SENIAT ante los contribuyentes}

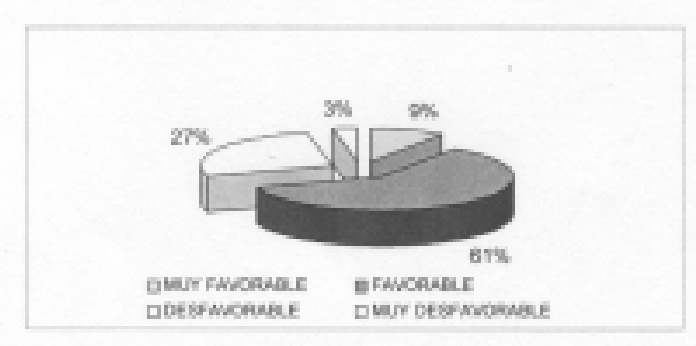

Paradójicamente a la opinión favorable que tiene la mayoría de los encuestados hacia el comportamiento de la actual administración tributaria, se observa todavía que un gran segmento de los contribuyentes muestra cierto grado de desconocimiento acerca de los cambios administrativos de la que ha sido objeto el órgano tributario; en relación con lo anterior, y tal como se muestra en el gráfico 2, los datos revelan que el 52\% de los encuestados poseen un bajo conocimiento sobre las reformas ocurridas en este sector del Estado, en cuanto a la simplificación administrativa de los trámites para el pago de los impuestos, desconocimiento que se hace más evidente en el sector comercio que el sector industrial, el cual parece estar más informado y actualizado en lo referente a los cambios acontecidos.

\section{Gráfico 2}

\section{Percepción del contribuyente sobre cambios en la} administración tributaria

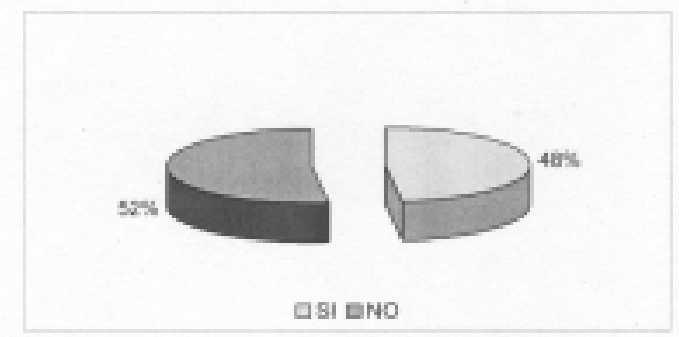


En el caso de aquellos contribuyentes que manifestaron tener un conocimiento sobre los cambios operados en la administración tributaria, a pesar de su enorme hermetismo (51\%), se pudo conocer la opinión sobre qué reformas son las más percibidas, el mayor porcentaje reconoce como cambio más notorio una mayor fiscalización del ente tributario, seguido de quienes resaltaron como significativo un aumento en la carga fiscal, mientras que otros, en menor proporción, consideraron que la atención personalizada es un nuevo rasgo dentro de la actual administración.

\section{Gráfico 3}

\section{Cambios en la administración tributaria percibidos por el contribuyente}

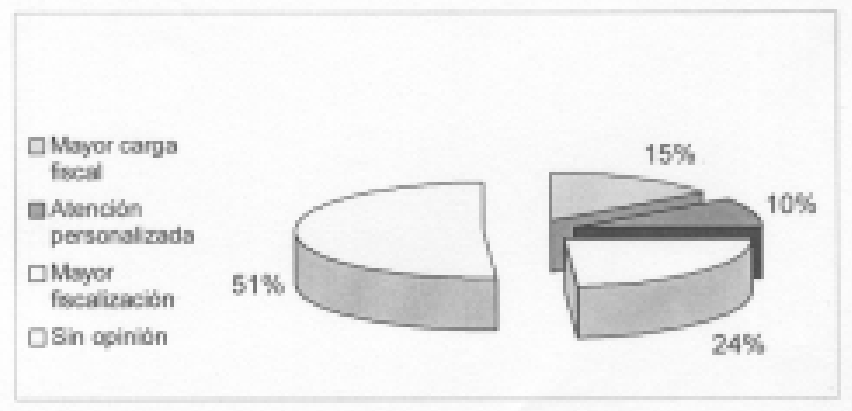

El enfoque económico neoinstitucional (North, 1993; Olson, 2001) ha subrayado la importancia que tiene el reconocimiento de las expectativas de los agentes económicos por parte de las autoridades estatales mediante el establecimiento de "contratos" o arreglos institucionales que sirvan como incentivos para generar un ambiente de confianza que procure un comportamiento adecuado dentro de las reglas de juego pactadas y, a su vez, debilite el desarrollo de actitudes depredadoras y egoístas entre los actores.

En el caso del SENIAT, la proyección de las expectativas de los contribuyentes que aparece en el gráfico 4, nos muestra a un agente económico que espera de su administración tributaria el otorgamiento de exoneraciones y descuentos por el cumplimiento oportuno de las obligaciones tributarias y una reducción de los montos de las multas en aquellos inclinados voluntariamente a ponerse a derecho con el pago de deudas. 


\section{Gráfico 4 \\ Expectativas de reconocimiento del contribuyente de parte de la administración tributaria}

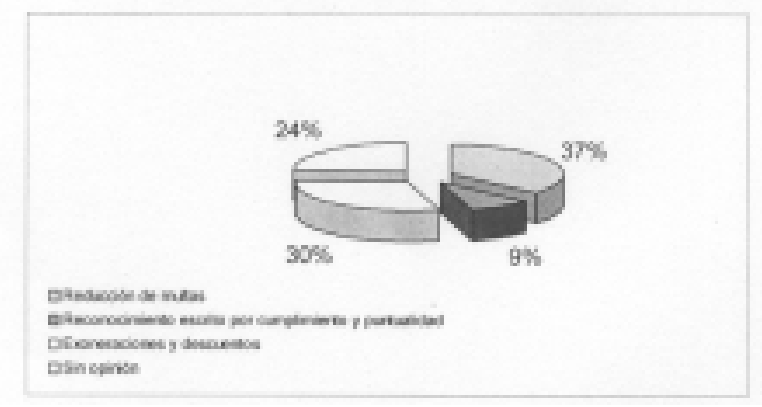

En cuanto al funcionamiento de la administración tributaria, las expectativas de los contribuyentes recaen principalmente en la aspiración de que el ente recaudador siga mejorando en la gestión de sus funciones básicas; sin embargo, lo que llama la atención, y que se ilustra en el gráfico 5, es el interés de un $24 \%$ de los encuestados sobre una mejor utilización de los recursos - obtenidos vía impuestos - en el mejoramiento de los servicios públicos.

\section{Gráfico 5}

Expectativas de los contribuyente en torno al SENIAT

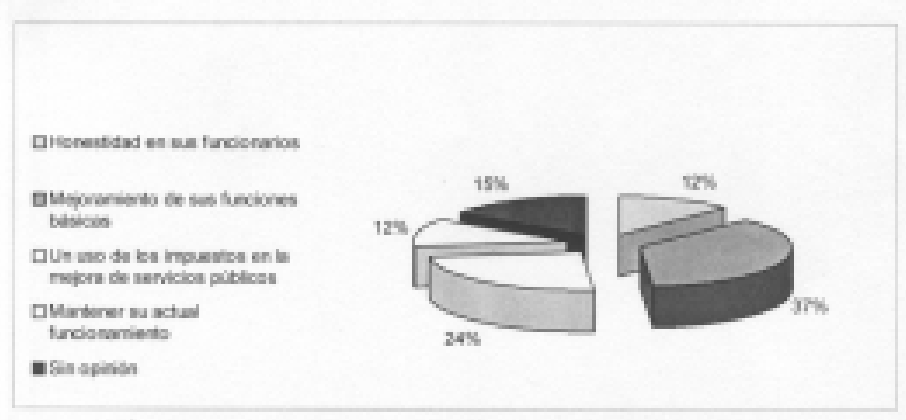

Mas allá de la imagen de eficiencia que podría acuñar progresivamente la administración tributaria venezolana con base en una mejora real de sus procedimientos, es importante reconocer que un factor que puede a la larga comprometer la legitimidad en la cancelación de los impuestos está asociado con el destino que se le den a dichos fondos provenientes de la recaudación. En torno a este asunto, la percepción 
de los contribuyentes presenta una imagen negativa del comportamiento del Estado en cuanto a su responsabilidad en la retribución de mejores servicios públicos como contraprestación por el pago de impuestos, lo cual se ve ratificado en que un $82 \%$ de los encuestados (véase gráfico 6) considera que el aumento de la recaudación no ha incidido directamente en una mejora significativa de los servicios públicos.

\section{Gráfico 6}

\section{Percepción en torno a la mejora de los servicios públicos con base al aumento de la recaudación}

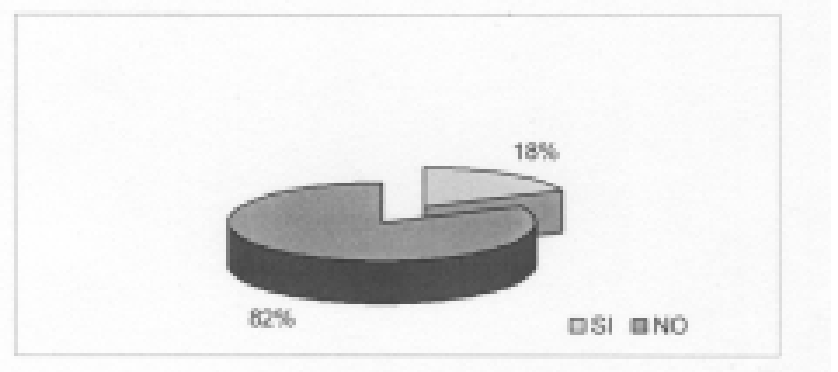

Si bien es cierto que el papel de la administración tributaria dentro de cualquier Estado es contribuir — mediante la recaudación de los impuestos - en la obtención de recursos fiscales para los gastos e inversiones que realiza dicho Estado, es necesario reconocer también que la forma y el destino que se le dé a tales recursos es un factor de enorme importancia para fortalecer la credibilidad y la confianza del contribuyente hacia el organismo recaudador. Esto último obedece a que en gran medida las bases de la legitimidad por rendimiento de esta agencia se encuentran situadas en otros circuitos - fundamentalmente de carácter decisorio y ejecutivo - dentro del entramado estatal.

\section{Conclusiones}

En el cumplimiento voluntario de las obligaciones tributarias de un contribuyente especial se debe considerar sus aspectos motivacionales con base en la percepción que tiene de la administración tributaria. En este sentido, la modernización del ente tributario ha contribuido en el desarrollo de una percepción positiva en este contribuyente en cuanto a las comodidades que ahora ofrecen los espacios físicos del organismo, la atención y trato de funcionarios calificados, así como la información por diversos medios para procurar la cultura hacia la tributación. 
En términos generales, se valora de manera satisfactoria en una proporción elevada de contribuyentes el mejoramiento de las funciones de la administración tributaria. No obstante, esta misma mayoría de contribuyentes especiales afirmaron que les gustaría el desarrollo de algún sistema de reconocimiento por parte del ente recaudador para aquellos que cumplan cabalmente en el tiempo con sus obligaciones fiscales. Se evidencia con esto la importancia que tiene en el contribuyente las formas de reconocimiento y distinción, como manera de resaltar la cooperación del contribuyente especial con el fisco.

Como se observó en este trabajo, el balance negativo en la percepción de los contribuyentes transciende los límites de funcionamiento de la propia administración, dado que él mismo gravita en torno a la relación entre los impuestos y la oferta de servicios públicos prestados por el Estado. Sin embargo, el hecho de que casi un 90\% de los contribuyentes especiales encuestados piense que la calidad de los servicios públicos recibidos no es adecuada en comparación al nivel de impuestos que se pagan y que alrededor de un $80 \%$ de estos mismos contribuyentes consideren que un aumento de la recaudación no mejorará significativamente los servicios públicos, nos lleva a preguntarnos: ¿qué incentivos puede tener el contribuyente hacia el cumplimiento voluntario de las obligaciones tributarias, cuando los servicios públicos prestados por el Estado son deficientes?

Más allá de las reformas administrativas del ente tributario mediante la incorporación de modernos sistemas para la recaudación, control e información al contribuyente y en los esfuerzos hechos en el aumento de la recaudación no se percibe satisfacción en el contribuyente con respecto a la calidad de vida y bienestar que debe llevar a los mismos — como incentivo - por cumplir con las obligaciones establecidas en la legislación vigente; al contrario, compromete seriamente el pago voluntario de los tributos.

Tales consideraciones hacen pensar que todavía se está muy lejos de lograr el desarrollo de una cultura tributaria basada en el cumplimiento voluntario de los impuestos por parte del gran contribuyente. A partir de la opinión de los encuestados se revela cierto consenso en torno a que el SENIAT pareciera no tomar en cuenta las expectativas de este contribuyente, limitándose la División de Contribuyentes Especiales sólo como una forma de organización para lograr un aumento de la recaudación a un menor costo a través de un mayor control y fiscalización de las operaciones del sector empresarial. 
Por último, es importante mencionar que esta estrategia de segmentación y diferenciación del universo de contribuyentes, en cuanto a definir a cierto grupo como especiales, expresa de forma obvia una alteración en el tratamiento igualitario del ciudadano contribuyente para la atención y estímulo al pago del tributo. La existencia de áreas exclusivas de la administración destinadas a la gestión tributaria de ciertos contribuyentes denominados como especiales refleja una diferencia en la condición del ciudadano contribuyente para ejercer sus deberes y derechos porque el resto de los contribuyentes - considerados ordinarios por la propia administración - no cuentan con las mismas facilidades y oportunidades de atención en cuanto al derecho a estar informado, escuchado y orientado de manera pronta y oportuna, lo que paradójicamente puede ir en perjuicio de la razón principal que ha segundado las reformas tributarias de los últimos años, la cual ha sido aumentar los niveles de recaudación a partir de una ampliación de la base imponible, que no es otra cosa que incrementar el mayor número de contribuyentes hasta donde más sea posible.

\section{Referencias bibliográficas}

BANCO MUNDIAL (1997), Informe sobre el desarrollo mundial. El Estado en un mundo en transformación, Washington, D.C.

CENTRO INTERAMERICANO DE ADMINISTRACIONES TRIBUTARIAS (1996), La administración de los grandes contribuyentes. Logros y perspectivas, Quito.

(2004), Estadísticas de ingresos tributarios del gobierno central, en www.ciat.org (fecha de consulta: 14 de octubre de 2005).

COMISIÓN DE ESTUDIO Y REFORMA FISCAL (1983), La reforma del sistema fiscal venezolano. Informe final, Caracas.

CONGRESO DE LA REPÚBLICA (1994), Código Orgánico Tributario, Caracas.

(1994), Ley de Impuesto al Consumo Suntuario y las Ventas al Mayor, Caracas.

INSTITUTO NACIONAL DE ESTADÍSTICA (2005), Estadísticas oficiales, en www.ine.gov.ve (fecha de consulta: 14 de octubre de 2005). 
METCALFE, Les y Sue Richards (1989), La modernización de la gestión pública, Instituto Nacional de Administración Pública, Madrid.

NORTH, Douglass (1993), Instituciones, cambio institucional y desempeño económico, Fondo de Cultura Económica, México.

OLSON, Mancur (2001), Poder y prosperidad, Siglo XXI, Argentina.

PURROY, Miguel (1990), "La urgente reforma tributaria", Revista SIC, Año LXX, No. 530, Caracas.

SERVICIO NACIONAL INTEGRADO DE ADMINISTRACIÓN ADUANERA Y TRIBUTARIA (2004), Banco de datos de Contribuyentes Especiales, Gerencia Regional de Tributos Internos del SENIAT, Región Zuliana, Maracaibo.

(2006), Resultados gestión de recaudación 2005, en www.seniat.gov.ve (Fecha de consulta: 24 de marzo de 2005).

TANZI, Vito (1996), "La reforma tributaria de América Latina en los últimos diez años", Revista de Política y Administración Tributaria No. 2, Ediciones SENIAT, Caracas. 